\title{
Proximal quasi-normal structure in convex metric spaces
}

\author{
Moosa Gabeleh
}

\begin{abstract}
We consider, in the setting of convex metric spaces, a new class of Kannan type cyclic orbital contractions, and study the existence of its best proximity points. The same problem is then discussed for relatively Kannan nonexpansive mappings, by using the concept of proximal quasinormal structure. In this way, we extend the main results in Abkar and Gabeleh [A. Abkar and M. Gabeleh, J. Nonlin. Convex Anal. 14 (2013), 653-659].
\end{abstract}

\section{Introduction}

Let $(X, d)$ be a metric space and $T: X \rightarrow X$ be a mapping. Call $T$, a Kannan contraction if there exists $\alpha \in\left[0, \frac{1}{2}\right)$ such that

$$
d(T x, T y) \leq \alpha[d(x, T x)+d(y, T y)],
$$

for all $x, y \in X$. We know that if $X$ is complete, then every Kannan contraction mapping has a unique fixed point, see [4]. In [9], Subrahmanyam proved that Kannan's fixed point theorem characterizes the metric completeness of underlying spaces, that is, a metric space $(X, d)$ is complete if and only if every Kannan contraction mapping on $X$ has a fixed point.

Key Words: Best proximity point; proximal quasi-normal structure; relatively Kannan nonexpansive mapping; convex metric space.

2010 Mathematics Subject Classification: 47H10, 47H09; 46B20.

Received: May, 2013

Revised: June, 2013.

Accepted: July, 2013. 
A mapping $T: X \rightarrow X$ is said to be Kannan nonexpansive, provided that

$$
d(T x, T y) \leq \frac{1}{2}[d(x, T x)+d(y, T y)]
$$

for all $x, y \in X$. Notice that mappings of the above type may or may not be nonexpansive in the usual case. In fact, the Kannan condition does not even imply continuity of the mapping. It was announced in [11] that $X$ is a Banach space with quasi-weak normal structure if and only if every Kannan nonexpansive mapping $T$ of a non-empty weakly compact convex subset $K$ of $X$ into itself has a fixed point.

Let us consider a mapping $T: A \cup B \rightarrow A \cup B$, where $A$ and $B$ are two nonempty subsets of a metric space $(X, d)$. The mapping $T$ is said to be a cyclic provided that $T(A) \subseteq B$ and $T(B) \subseteq A$. If $A \cap B=\emptyset$, then a cyclic map cannot have fixed points. In this case, it is interesting to study the existence of best proximity points $p \in A \cup B$, characterized as

$$
d(p, T p)=\operatorname{dist}(A, B):=\inf \{d(x, y):(x, y) \in A \times B\} .
$$

The relevance of best proximity points is that they provide optimal solutions for the problem of best approximation between two sets.

Eldred, Kirk and Veeramani [2] established the existence of a best proximity point for cyclic relatively nonexpansive mappings by using a geometric notion of proximal normal structure in the setting of Banach spaces.

Theorem 1.1.([2]) Let $(A, B)$ be a nonempty, weakly compact and convex pair in a Banach space $X$. Let $T: A \cup B \rightarrow A \cup B$ be a cyclic relatively nonexpansive mapping, that is, $T$ is cyclic and $\|T x-T y\| \leq\|x-y\|$ for each $(x, y) \in A \times B$. Suppose that $(A, B)$ has the proximal normal structure. Then $T$ has a best proximity point.

After that in [7], the authors established the existence of best proximity points for cyclic relatively nonexpansive mappings without invoking proximal normal structure.

The notion of weak cyclic Kannan contractions was introduced in [5] as follows.

Definition 1.2. Let $A, B$ be a two nonempty subsets of a metric space $(X, d)$. A mapping $T: A \cup B \rightarrow A \cup B$ is said to be a weak cyclic Kannan contraction mapping if $T$ is cyclic and satisfies the following condition.

$$
d(T x, T y) \leq \alpha\{d(x, T x)+d(y, T y)\}+(1-2 \alpha) \operatorname{dist}(A, B),
$$


for some $\alpha \in\left(0, \frac{1}{2}\right)$ and for all $(x, y) \in A \times B$.

If in above definition $\alpha=\frac{1}{2}$, we say that $T$ is relatively Kannan nonexpansive, that is, $T$ is cyclic on $A \cup B$ and satisfies the following condition.

$$
d(T x, T y) \leq \frac{1}{2}\{d(x, T x)+d(y, T y)\}, \forall(x, y) \in A \times B .
$$

The following theorem guarantees the existence, uniqueness and convergence of a best proximity point for weak cyclic Kannan contractions in the setting of uniformly convex Banach spaces.

Theorem 1.3.([5]) Let $(A, B)$ be a nonempty closed convex pair in a uniformly convex Banach space $X$. Suppose that $T: A \cup B \rightarrow A \cup B$ is a weak cyclic Kannan contraction mapping. Then $T$ has a unique best proximity point $z \in A$. Moreover, the sequence $\left\{T^{2 n} x\right\}$ converges to $z$ for any $x \in A$.

Note that the geometric property of uniformly convexity of a Banach space $X$, plays an important role in the proof of Theorem 1.3. In [1], the authors established the next existence result of best proximity points for weak cyclic Kannan contractions in Banach spaces without uniformly convexity.

Theorem 1.4.([1]) Let $(A, B)$ be a nonempty weakly compact convex pair in a Banach space $X$. Assume that $T: A \cup B \rightarrow A \cup B$ is a weak cyclic Kannan contraction mapping. Then $T$ has a best proximity point.

Another notion of cyclic mappings was introduced in [3] as below.

Definition 1.5.([3]) Let $(A, B)$ be a nonempty pair of subsets of a Banach space $X$. A mapping $T: A \cup B \rightarrow A \cup B$ is said to be a relatively u-continuous mapping if $T$ is cyclic on $A \cup B$ and satisfies the following condition.

$$
\forall \varepsilon>0, \exists \delta>0 ; \text { if }\|x-y\|<\delta+\operatorname{dist}(A, B) \text { then }\|T x-T y\|<\varepsilon+\operatorname{dist}(A, B),
$$

for all $(x, y) \in A \times B$.

It is clear that every cyclic relatively nonexpansive mapping is a cyclic relatively $\mathrm{u}$-continuous. The following best proximity point theorem is the main result of [3].

Theorem 1.6.([3]) Let $(A, B)$ be a nonempty compact convex pair of subsets of a strictly convex Banach space $X$ and $T: A \cup B \rightarrow A \cup B$ be a relatively u-continuous mapping. Then there exists $\left(x_{0}, y_{0}\right) \in A \times B$ such that 
$\left\|x_{0}-T x_{0}\right\|=\left\|T y_{0}-y_{0}\right\|=\operatorname{dist}(A, B)$.

In the current paper, we introduce a new class of cyclic mappings, called cyclic orbital contractions in the sense of Kannan, which contains the class of weak cyclic contractions as a subclass. We prove a similar result of Theorem 1.4 for this class of mappings in the setting of convex metric spaces. Moreover, we prove a best proximity point theorem for relatively Kannan nonexpansive mappings which are u-continuous by using a geometric notion of proximal quasi-normal structure.

\section{Preliminaries}

In [10], Takahashi introduced the notion of convexity in metric spaces as follows.

Definition 2.1. Let $(X, d)$ be a metric space and $I:=[0,1]$. A mapping $\mathcal{W}: X \times X \times I \rightarrow X$ is said to be a convex structure on $X$ provided that for each $(x, y ; \lambda) \in X \times X \times I$ and $u \in X$,

$$
d(u, \mathcal{W}(x, y ; \lambda)) \leq \lambda d(u, x)+(1-\lambda) d(u, y) .
$$

A metric space $(X, d)$ together with a convex structure $\mathcal{W}$ is called a convex metric space, which is denoted by $(X, d, \mathcal{W})$. A Banach space and each of its convex subsets are convex metric spaces. But a Frechet space is not necessary a convex metric space. The other examples of convex metric spaces which are not imbedded in any Banach space can be founded in [10].

To describe our results, we need some definitions and preliminary facts from the references [2] and [10].

Definition 2.2. A subset $K$ of a convex metric space $(X, d, \mathcal{W})$ is said to be a convex set provided that $\mathcal{W}(x, y ; \lambda) \in K$ for all $x, y \in K$ and $\lambda \in I$.

Proposition 2.3. Let $(X, d, \mathcal{W})$ be a convex metric space and let $B(x ; r)$ denote the closed ball centered at $x \in X$ with radius $r \geq 0$. Then $B(x ; r)$ is a convex subset of $X$.

Proposition 2.4. Let $\left\{K_{\alpha}\right\}_{\alpha \in A}$ be a family of convex subsets of $X$, then $\bigcap_{\alpha \in A} K_{\alpha}$ is also a convex subset of $X$.

Definition 2.5. A convex metric space $(X, d, \mathcal{W})$ is said to have property $(\mathrm{C})$ if every bounded decreasing net of nonempty closed convex subsets of $X$ has 
a nonempty intersection.

For example every weakly compact convex subset of a Banach space has property $(\mathrm{C})$. The next example ensures that condition $(\mathrm{C})$ is natural as well in the metrical setting.

Example 2.6. ([8]) Let $\mathcal{H}$ be a Hilbert space and let $X$ be a nonempty closed subset of $\{x \in \mathcal{H}:\|x\|=1\}$ such that if $x, y \in X$ and $\alpha, \beta \in[0,1]$ with $\alpha+\beta=1$, then $\frac{\alpha x+\beta y}{\|\alpha x+\beta y\|} \in X$ and $\operatorname{diam}(X) \leq \frac{\sqrt{2}}{2}$, where $\operatorname{diam}(X):=$ $\sup \{d(x, y): x, y \in X\}$. Let $\left.d(x, y):=\cos ^{-1}(<x, y\rangle\right)$ for all $x, y \in X$, where $\langle$,$\rangle is the inner product of \mathcal{H}$. If we define the convex structure $\mathcal{W}: X \times X \times I \rightarrow X$ with $\mathcal{W}(x, y, \lambda):=\frac{\lambda x+(1-\lambda) y}{\|\lambda x+(1-\lambda) y\|}$, then $(X, d)$ is a complete convex metric space which has the property $(\mathrm{C})$ (for more information see Example 2 of [8]).

Let $A$ and $B$ be two nonempty subsets of a convex metric space $(X, d, \mathcal{W})$. We shall say that a pair $(A, B)$ in a convex metric space $(X, d, \mathcal{W})$ satisfies a property if both $A$ and $B$ satisfy that property. For instance, $(A, B)$ is convex if and only if both $A$ and $B$ are convex; $(A, B) \subseteq(C, D) \Leftrightarrow A \subseteq C$, and $B \subseteq D$. We shall also adopt the following notations.

$$
\begin{aligned}
\delta_{x}(A) & :=\sup \{d(x, y): y \in A\} \text { for all } x \in X, \\
\delta(A, B) & :=\sup \{d(x, y): x \in A, y \in B\} \\
\operatorname{diam}(A) & :=\delta(A, A) .
\end{aligned}
$$

The closed and convex hull of a set $A$ will be denoted by $\overline{\operatorname{con}}(A)$ and defined as below.

$\overline{\operatorname{con}}(A):=\bigcap\{C: C$ is a closed and convex subset of $X$ such that $C \supseteq A\}$.

The pair $(x, y) \in A \times B$ is said to be proximal in $(A, B)$ if $d(x, y)=\operatorname{dist}(A, B)$. Moreover, we set

$$
\begin{aligned}
& A_{0}:=\left\{x \in A: d\left(x, y^{\prime}\right)=\operatorname{dist}(A, B), \text { for some } y^{\prime} \in B\right\}, \\
& B_{0}:=\left\{y \in B: d\left(x^{\prime}, y\right)=\operatorname{dist}(A, B), \text { for some } x^{\prime} \in A\right\} .
\end{aligned}
$$

We note that if $(A, B)$ is a nonempty weakly compact and convex pair of subsets of a Banach space $X$, then also is the pair $\left(A_{0}, B_{0}\right)$ and it is easy to see that $\operatorname{dist}(A, B)=\operatorname{dist}\left(A_{0}, B_{0}\right)$.

For a cyclic mapping $T: A \cup B \rightarrow A \cup B$ and $x \in A \cup B$, we define the orbit setting at $x$ by

$$
\mathcal{O}_{T} x:=\left\{T x, T^{3} x, \ldots, T^{2 n-1} x, \ldots\right\},
$$


where, for each $n \geq 1, T^{n}$ stands for the n-th iterate of $T$. Note that if $(x, y) \in A \times B$, then $\mathcal{O}_{T} x \subseteq B$ and $\mathcal{O}_{T} y \subseteq A$.

Definition 2.7. Let $A$ be a nonempty subset in a metric space $(X, d)$. A point $p$ in $A$ is said to be a diametral point if $\delta_{p}(A)=\operatorname{diam}(A)$.

Definition 2.8. A convex metric space $(X, d, \mathcal{W})$ is said to have normal structure if for each bounded, closed and convex subset $E$ of $X$ which contains at least two points, there exists an element $p \in E$ which is a nondiametral point.

Here, we recall the geometric notion of proximal normal structure which was introduced in [2].

Definition 2.9. A pair $(A, B)$ of subsets of a linear space $X$ is said to be a proximal pair if for each $(x, y) \in A \times B$ there exists $\left(x^{\prime}, y^{\prime}\right) \in A \times B$ such that

$$
\left\|x-y^{\prime}\right\|=\left\|x^{\prime}-y\right\|=\operatorname{dist}(A, B) .
$$

Definition 2.10. A convex pair $\left(K_{1}, K_{2}\right)$ in a Banach space $X$ is said to have proximal normal structure if for any bounded, closed and convex proximal pair $\left(H_{1}, H_{2}\right) \subseteq\left(K_{1}, K_{2}\right)$ for which $\operatorname{dist}\left(H_{1}, H_{2}\right)=\operatorname{dist}\left(K_{1}, K_{2}\right)$ and $\delta\left(H_{1}, H_{2}\right)>$ $\operatorname{dist}\left(H_{1}, H_{2}\right)$, there exits $\left(x_{1}, x_{2}\right) \in H_{1} \times H_{2}$ such that

$$
\delta_{x_{1}}\left(H_{2}\right)<\delta\left(H_{1}, H_{2}\right), \quad \delta_{x_{2}}\left(H_{1}\right)<\delta\left(H_{1}, H_{2}\right) .
$$

It was announced in [2] that every nonempty, bounded, closed and convex pair of subsets of a uniformly convex Banach space $X$ has a proximal normal structure (Proposition 2.1 of [2]).

\section{Cyclic orbital contractions in the sense of Kannan}

We begin our main result of this section with the following definition.

Definition 3.1. Let $(A, B)$ be a nonempty pair of subsets of a metric space $(X, d)$. A mapping $T: A \cup B \rightarrow A \cup B$ is said to be a cyclic orbital contraction in the sense of Kannan if $T$ is cyclic on $A \cup B$ and there exists $\alpha \in\left(0, \frac{1}{2}\right)$ such that

$$
d(T x, T y) \leq \alpha\left\{\delta_{x}\left(\mathcal{O}_{T} x\right)+\delta_{y}\left(\mathcal{O}_{T} y\right)\right\}+(1-2 \alpha) \operatorname{dist}(A, B),
$$

for all $(x, y) \in A \times B$. 
It is clear that every weak cyclic Kannan contraction is cyclic orbital contraction in the sense of Kannan. Let us state the following theorem which ensures the existence of best proximity points for a cyclic orbital contraction in the sense of Kannan.

Theorem 3.2. Let $(A, B)$ be a nonempty, bounded, closed and convex pair in a convex metric space $(X, d, \mathcal{W})$. Suppose that $T: A \cup B \rightarrow A \cup B$ is a cyclic orbital contraction in the sense of Kannan. If $X$ has the property (C), then $T$ has a best proximity point.

Proof. Let $\Sigma$ denote the set of all nonempty, bounded, closed and convex pairs $(E, F)$ which are subsets of $(A, B)$ and such that $T$ is cyclic on $E \cup F$. Note that $(A, B) \in \Sigma$. Also, $\Sigma$ is partially ordered by the reverse inclusion, that is $\left(E_{1}, F_{1}\right) \leq\left(E_{2}, F_{2}\right) \Leftrightarrow\left(E_{2}, F_{2}\right) \subseteq\left(E_{1}, F_{1}\right)$. By the fact that $X$ has the property $(\mathrm{C})$, every increasing chain in $\Sigma$ is bounded above. So, by using Zorn's lemma we obtain a minimal element say $(C, D) \in \Sigma$. We note that $(\overline{c o n}(T(D)), \overline{c o n}(T(C)))$ is a nonempty, bounded, closed and convex pair in $X$ and $(\overline{c o n}(T(D)), \overline{c o n}(T(C))) \subseteq(C, D)$. Further,

$$
T(\overline{c o n}(T(D))) \subseteq T(C) \subseteq \overline{c o n}(T(C)),
$$

and also,

$$
T(\overline{c o n}(T(C))) \subseteq \overline{c o n}(T(D)),
$$

that is, $T$ is cyclic on $\overline{c o n}(T(D)) \cup \overline{c o n}(T(C))$. It now follows from the minimality of $(C, D)$ that

$$
\overline{\operatorname{con}}(T(D))=C, \overline{\operatorname{con}}(T(C))=D .
$$

Let $x \in C$, then $D \subseteq B\left(x ; \delta_{x}(D)\right)$. Now, if $y \in D$ we have

$$
\begin{aligned}
d(T x, T y) & \leq \alpha\left\{\delta_{x}\left(\mathcal{O}_{T} x\right)+\delta_{y}\left(\mathcal{O}_{T} y\right)\right\}+(1-2 \alpha) \operatorname{dist}(A, B) \\
& \leq 2 \alpha \delta(C, D)+(1-2 \alpha) \operatorname{dist}(A, B) .
\end{aligned}
$$

Hence, for all $y \in D$ we have

$$
T y \in B(T x ; 2 \alpha \delta(C, D)+(1-2 \alpha) \operatorname{dist}(A, B)),
$$

and so,

$$
T(D) \subseteq B(T x ; 2 \alpha \delta(C, D)+(1-2 \alpha) \operatorname{dist}(A, B)) .
$$

Thus,

$$
C=\overline{c o n}(T(D)) \subseteq B(T x ; 2 \alpha \delta(C, D)+(1-2 \alpha) \operatorname{dist}(A, B)) .
$$


Therefore,

$$
d(z, T x) \leq 2 \alpha \delta(C, D)+(1-2 \alpha) \operatorname{dist}(A, B), \quad \forall z \in C .
$$

This implies that

$$
\delta_{T x}(C) \leq 2 \alpha \delta(C, D)+(1-2 \alpha) \operatorname{dist}(A, B) .
$$

Similarly, if $y \in D$, we conclude that

$$
\delta_{T y}(D) \leq 2 \alpha \delta(C, D)+(1-2 \alpha) \operatorname{dist}(A, B) .
$$

If we put

$$
\begin{aligned}
& E:=\left\{x \in C: \delta_{x}(D) \leq 2 \alpha \delta(C, D)+(1-2 \alpha) \operatorname{dist}(A, B)\right\}, \\
& F:=\left\{y \in D: \delta_{y}(C) \leq 2 \alpha \delta(C, D)+(1-2 \alpha) \operatorname{dist}(A, B)\right\},
\end{aligned}
$$

then $T(D) \subseteq E$ and $T(C) \subseteq F$. Besides, it is easy to check that

$$
\begin{aligned}
& E=\bigcap_{y \in D} B(y ; 2 \alpha \delta(C, D)+(1-2 \alpha) \operatorname{dist}(A, B)) \cap C, \\
& F=\bigcap_{x \in C} B(x ; 2 \alpha \delta(C, D)+(1-2 \alpha) \operatorname{dist}(A, B)) \cap D .
\end{aligned}
$$

Further, if $x \in E$ then by $(7), T x \in F$, i.e. $T\left(E_{1}\right) \subseteq E_{2}$ and also by the relation (8) $T(F) \subseteq E$, that is, $T$ is cyclic on $E \cup F$. It now follows from the minimality of $(C, D)$ that $E=C$ and $F=D$. This deduces that

$$
\delta_{x}(D) \leq 2 \alpha \delta(C, D)+(1-2 \alpha) \operatorname{dist}(A, B), \quad \forall x \in C .
$$

We have

$$
\delta(C, D)=\sup _{x \in C} \delta_{x}(D) \leq 2 \alpha \delta(C, D)+(1-2 \alpha) \operatorname{dist}(A, B),
$$

and then $\delta(C, D)=\operatorname{dist}(A, B)$. Therefore, for each pair $\left(x^{*}, y^{*}\right) \in C \times D$ we must have

$$
d\left(x^{*}, T x^{*}\right)=d\left(T y^{*}, y^{*}\right)=\operatorname{dist}(A, B) .
$$

The following corollary ensures the existence and uniqueness of a best proximity pair in the setting of Banach spaces.

Corollary 3.3. Let $(A, B)$ be a nonempty, bounded, closed and convex pair in a reflexive and strictly convex Banach space $X$. Suppose that $T: A \cup B \rightarrow$ $A \cup B$ is a cyclic orbital contraction in the sense of Kannan. Then there exists a unique pair $\left(x^{*}, y^{*}\right) \in A \times B$ such that $\left\|x^{*}-T x^{*}\right\|=\left\|T y^{*}-y^{*}\right\|=\operatorname{dist}(A, B)$. 


\section{Relatively Kannan nonexpansive mappings}

In this section we investigate the existence of a best proximity point for relatively Kannan nonexpansive mappings in the setting of convex metric spaces. In this order, we need a geometric notion of proximal quasi-normal structure for a convex pair of subsets of a convex metric space.

Definition 4.1.([1]) A convex pair $\left(K_{1}, K_{2}\right)$ in a convex metric space $(X, d, \mathcal{W})$ is said to have proximal quasi-normal structure if for any bounded, closed and convex proximal pair $\left(H_{1}, H_{2}\right) \subseteq\left(K_{1}, K_{2}\right)$ for which $\operatorname{dist}\left(H_{1}, H_{2}\right)=$ $\operatorname{dist}\left(K_{1}, K_{2}\right)$ and $\delta\left(H_{1}, H_{2}\right)>\operatorname{dist}\left(H_{1}, H_{2}\right)$, there exits $(u, v) \in H_{1} \times H_{2}$ such that

$$
d(u, y)<\delta\left(H_{1}, H_{2}\right), \quad d(x, v)<\delta\left(H_{1}, H_{2}\right),
$$

for all $(x, y) \in H_{1} \times H_{2}$.

Note that if the convex proximal pair $\left(K_{1}, K_{2}\right)$ of a convex metric space $X$ has proximal normal structure, then $\left(K_{1}, K_{2}\right)$ has proximal quasi-normal structure.

Definition 4.2. $([6])$ Let $(A, B)$ be a nonempty pair of subsets of a metric space $(X, d)$. We say that the pair $(A, B)$ is proximal compactness provided that every net $\left(\left\{x_{\alpha}\right\},\left\{y_{\alpha}\right\}\right)$ of $A \times B$ satisfying the condition that $d\left(x_{\alpha}, y_{\alpha}\right) \rightarrow \operatorname{dist}(A, B)$, has a convergent subnet in $A \times B$.

It is clear that if $(A, B)$ is a compact pair in a metric space $(X, d)$ then $(A, B)$ is proximal compactness. The next lemma will be used in our main theorem of this section.

Lemma 4.3.([1]) Let $\left(K_{1}, K_{2}\right)$ be a nonempty pair of a convex metric space $(X, d, \mathcal{W})$. Then $\delta\left(K_{1}, K_{2}\right)=\delta\left(\overline{c o n}\left(K_{1}\right), \overline{c o n}\left(K_{2}\right)\right)$.

Next, we shall prove our main result of this section.

Theorem 4.4. Let $(A, B)$ be a nonempty, bounded, closed and convex pair in a convex metric space $(X, d, \mathcal{W})$ such that $A_{0}$ is nonempty and $(A, B)$ is proximal compactness. Suppose that $T: A \cup B \rightarrow A \cup B$ is a relatively Kannan nonexpansive mapping and $(A, B)$ has the proximal quasi-normal structure. Moreover, let $T$ be a relatively u-continuous and $X$ has the property (C). Then $T$ has a best proximity point.

Proof. Let $\Sigma$ denote the set of all nonempty, closed and convex pairs $(E, F)$ 
which are subsets of $(A, B)$ and such that $T$ is cyclic on $E \cup F$ and $d(x, y)=$ $\operatorname{dist}(A, B)$ for some $(x, y) \in E \times F$. Note that $(A, B) \in \Sigma$ by the fact that $A_{0}$ is nonempty. Also, $\Sigma$ is partially ordered by the reverse inclusion. Assume that $\left\{\left(E_{\alpha}, F_{\alpha}\right)\right\}_{\alpha}$ is a descending chain in $\Sigma$. Set $E:=\bigcap E_{\alpha}$ and $F:=\bigcap F_{\alpha}$. Since $X$ has the property $(\mathrm{C})$, we conclude that $(E, F)$ is a nonempty pair. Also, by Proposition 2.4, $(E, F)$ is a closed and convex pair. Moreover,

$$
T(E)=T\left(\bigcap E_{\alpha}\right) \subseteq \bigcap T\left(E_{\alpha}\right) \subseteq \bigcap F_{\alpha}=F
$$

Similarly, we can see that $T(F) \subseteq E$, that is, $T$ is cyclic on $E \cup F$. Now, let $\left(x_{\alpha}, y_{\alpha}\right) \in E_{\alpha} \times F_{\alpha}$ be such that $d\left(x_{\alpha}, y_{\alpha}\right)=\operatorname{dist}(A, B)$. Since $(A, B)$ is proximal compactness, $\left(x_{\alpha}, y_{\alpha}\right)$ has a convergent subsequence say $\left(x_{\alpha_{i}}, y_{\alpha_{i}}\right)$ such that $x_{\alpha_{i}} \rightarrow x \in A$ and $y_{\alpha_{i}} \rightarrow y \in B$. Thus,

$$
d(x, y)=\lim _{i} d\left(x_{\alpha_{i}}, y_{\alpha_{i}}\right)=\operatorname{dist}(A, B)
$$

Therefore, there exists an element $(x, y) \in E \times F$ such that $d(x, y)=\operatorname{dist}(A, B)$. Hence, every increasing chain in $\Sigma$ is bounded above with respect to reverse inclusion relation. Then by using Zorn's Lemma we can get a minimal element say $\left(K_{1}, K_{2}\right)$. Let $r>0$ be such that $r \geq \operatorname{dist}(A, B)$ and consider $\left(x^{*}, y^{*}\right) \in K \times K_{2}$ such that

$$
d\left(x^{*}, y^{*}\right)=\operatorname{dist}(A, B), d\left(x^{*}, T x^{*}\right) \leq r \text { and } d\left(T y^{*}, y^{*}\right) \leq r .
$$

Set,

$$
K_{1}^{r}=\left\{x \in K_{1}: d(x, T x) \leq r\right\}, K_{2}^{r}=\left\{x \in K_{2}: d(T x, x) \leq r\right\}
$$

and put $C_{1}^{r}:=\overline{c o n}\left(T\left(K_{1}^{r}\right)\right), C_{2}^{r}:=\overline{c o n}\left(T\left(K_{2}^{r}\right)\right)$. We prove that $T$ is cyclic on $C_{1}^{r} \cup C_{2}^{r}$. At first, we show that $C_{1}^{r} \subseteq K_{2}^{r}$. Let $x \in C_{1}^{r}$. If $d(T x, x)=\operatorname{dist}(A, B)$, then $x \in K_{2}^{r}$. Let $d(T x, x)>\operatorname{dist}(A, B)$. Put, $h:=\sup \left\{d(T z, T x): z \in K_{1}^{r}\right\}$. We note that $B(T x ; h) \supseteq T\left(K_{1}^{r}\right)$, that is, $C_{1}^{r} \subseteq B(T x ; h)$. Since $x \in C_{1}^{r}$, we have $d(T x, x) \leq h$. It now follows from the definition of $h$ that for each $\varepsilon>0$ there exists $z \in K_{1}^{r}$ such that $h-\varepsilon \leq d(T z, T x)$. Therefore,

$$
\begin{gathered}
d(T x, x)-\varepsilon \leq h-\varepsilon \leq d(T z, T x) \\
\leq \frac{1}{2}[d(z, T z)+d(T x, x)] \leq \frac{1}{2} d(T x, x)+\frac{1}{2} r .
\end{gathered}
$$

Hence, $d(T x, x) \leq r+2 \varepsilon$ and so, $x \in K_{2}^{r}$. Thus, $C_{1}^{r} \subseteq K_{2}^{r}$. This implies that

$$
T\left(C_{1}^{r}\right) \subseteq T\left(K_{2}^{r}\right) \subseteq \overline{c o n}\left(T\left(K_{2}^{r}\right)\right)=C_{2}^{r} .
$$


Similarly, we can see that $T\left(C_{2}^{r}\right) \subseteq C_{1}^{r}$, that is, $T$ is cyclic on $C_{1}^{r} \cup C_{2}^{r}$. We now claim that $\delta\left(C_{1}^{r}, C_{2}^{r}\right) \leq r$. By using Lemma 4.3 we have

$$
\begin{gathered}
\delta\left(C_{1}^{r}, C_{2}^{r}\right)=\delta\left(\overline{\operatorname{con}}\left(T\left(K_{1}^{r}\right), \overline{\operatorname{con}}\left(T\left(K_{2}^{r}\right)\right)\right)\right. \\
=\delta\left(T\left(K_{1}^{r}\right), T\left(K_{2}^{r}\right)\right)=\sup \left\{d(T x, T y): x \in K_{1}^{r}, y \in K_{2}^{r}\right\} \\
\leq \sup \left\{\frac{1}{2}[d(x, T x)+d(T y, y)]: x \in K_{1}^{r}, y \in K_{2}^{r}\right\} \\
\leq \frac{1}{2}[r+r]=r .
\end{gathered}
$$

Besides, since $\left(x^{*}, y^{*}\right) \in K_{1} \times K_{2}$ and $d\left(x^{*}, y^{*}\right)=\operatorname{dist}(A, B)$, for any $\zeta>0$, we have $d\left(x^{*}, y^{*}\right)<\zeta+\operatorname{dist}(A, B)$. By the fact that $T$ is relatively u-continuous map,

$$
\operatorname{dist}(A, B) \leq d\left(T x^{*}, T y^{*}\right)<\varepsilon+\operatorname{dist}(A, B),
$$

for each $\varepsilon>0$. This implies that $d\left(T x^{*}, T y^{*}\right)=\operatorname{dist}(A, B)$ and so, $\operatorname{dist}\left(C_{2}^{r}, C_{1}^{r}\right)=\operatorname{dist}(A, B)$. Let

$$
r_{0}:=\inf \left\{d(x, T x): x \in K_{1} \cup K_{2}\right\} .
$$

Then $r_{0} \geq \operatorname{dist}(A, B)$. Let $\left\{r_{n}\right\}$ be a nonnegative sequence such that $r_{n} \downarrow r_{0}$. Thus, $\left(\left\{C_{1}^{r_{n}}\right\},\left\{C_{2}^{r_{n}}\right\}\right)$ are descending sequences of nonempty, bounded, closed and convex subsets of $\left(K_{1}, K_{2}\right)$. Since $X$ has the property (C),

$$
C_{1}^{r_{0}}=\bigcap_{n=1}^{\infty} C_{1}^{r_{n}} \neq \emptyset, C_{2}^{r_{0}}=\bigcap_{n=1}^{\infty} C_{2}^{r_{n}} \neq \emptyset .
$$

Moreover, by the preceding argument, $T: C_{1}^{r_{0}} \cup C_{2}^{r_{0}} \rightarrow C_{1}^{r_{0}} \cup C_{2}^{r_{0}}$ is a cyclic mapping. Further, since $\operatorname{dist}\left(C_{2}^{r_{n}}, C_{1}^{r_{n}}\right)=\operatorname{dist}(A, B)$ for all $n \in \mathbb{N}$, we deduce that $\operatorname{dist}\left(C_{2}^{r_{0}}, C_{1}^{r_{0}}\right)=\operatorname{dist}(A, B)$. It now follows from the minimality of $\left(K_{1}, K_{2}\right)$ that $C_{2}^{r_{0}}=K_{1}$ and $C_{1}^{r_{0}}=K_{2}$. Therefore, $d(x, T x) \leq r_{0}$ for all $x \in K_{1} \cup K_{2}$. Assume that $r_{0}>\operatorname{dist}(A, B)$. Since the pair $(A, B)$ has proximal quasi-normal structure, there exists $(u, v) \in K_{1} \times K_{2}$ such that

$$
d(u, y)<\delta\left(K_{1}, K_{2}\right) \leq r_{0}, d(x, v)<\delta\left(K_{1}, K_{2}\right) \leq r_{0},
$$

for all $(x, y) \in K_{1} \times K_{2}$. Hence,

$$
d(u, T u)<\delta\left(K_{1}, K_{2}\right) \leq r_{0} \& d(T v, v)<\delta\left(K_{1}, K_{2}\right) \leq r_{0} .
$$

This is a contradiction, that is, $r_{0}=\operatorname{dist}(A, B)$ and so,

$$
d(x, T x)=d(y, T y)=\operatorname{dist}(A, B),
$$

for all $(x, y) \in K_{1} \times K_{2}$. This completes the proof. 
As a result of Theorem 4.4, we obtain the next corollary which is the main result of $[1]$.

Theorem 4.5.([1]) Let $(A, B)$ be a nonempty, weakly compact convex pair in a Banach space $X$ and suppose $(A, B)$ has proximal quasi-normal structure. Let $T: A \cup B \rightarrow A \cup B$ be a cyclic mapping such that

$$
\|T x-T y\| \leq \min \left\{\frac{1}{2}(\|x-T x\|+\|y-T y\|),\|x-y\|\right\},
$$

for all $(x, y) \in A \times B$. Then there exists $\left(x^{*}, y^{*}\right) \in A \times B$ such that

$$
\left\|x^{*}-T x^{*}\right\|=\left\|T y^{*}-y^{*}\right\|=\operatorname{dist}(A, B) .
$$

Let us illustrate Theorem 4.4 with the following example.

Example 4.5. Let $X:=[-1,1]$ and define a metric $d$ on $X$ by

$$
d(x, y)=\left\{\begin{array}{l}
0, \quad \text { if } x=y, \\
\max \{|x|,|y|\}, \quad \text { if } x \neq y .
\end{array}\right.
$$

Define $\mathcal{W}: X \times X \times I \rightarrow X$ with

$$
\mathcal{W}(x, y, \lambda)=\lambda \min \{|x|,|y|\},
$$

for each $x, y \in X$ and $\lambda \in I$. We show that $\mathcal{W}$ is a convex stricture on $X$. Let $x, y \in X$ and $\lambda \in I$. We may assume that $|x| \leq|y|$. Then for each $u \in X$ we have

$$
\begin{gathered}
d(u, \mathcal{W}(x, y, \lambda))=\max \{|u|, \lambda \min \{|x|,|y|\}\} \\
=\max \{|u|, \lambda|x|\} \leq \max \{|u|,|x|\} \\
=\lambda \max \{|u|,|x|\}+(1-\lambda) \max \{|u|,|x|\} \\
\leq \lambda \max \{|u|,|x|\}+(1-\lambda) \max \{|u|,|y|\} \\
=\lambda d(u, x)+(1-\lambda) d(u, y) .
\end{gathered}
$$

This implies that $(X, d, \mathcal{W})$ is a convex metric space. Now, let $E$ be a nonempty convex subset of $X$. Then $\mathcal{W}(x, y, \lambda) \in E$ for each $x, y \in E$ and $\lambda \in I$. If $\lambda=0$, then we conclude that $0 \in E$. Therefore, the convex metric space $(X, d, \mathcal{W})$ must be have the property $(\mathrm{C})$. Suppose that $A:=[0,1]$. Thus, $A$ is a bounded closed and convex subset of $X$. Note that every convergent sequence in this metric space converges to $0 \in A$. Let $T: A \rightarrow A$ be a mapping defined as

$$
T x= \begin{cases}0, & \text { if } x=1, \\ \frac{1}{2}, & \text { if } x \neq 1 .\end{cases}
$$


We claim that $T$ is relatively Kannan nonexpansive. For this purpose it is sufficient to consider $x=1$ and $y \neq 1$. Then $d(T x, T y)=\frac{1}{2}, d(x, T x)=1$ and $d(y, T y)=\max \left\{y, \frac{1}{2}\right\}$. Hence,

$$
d(T x, T y) \leq \frac{1}{2}[d(x, T x)+d(y, T y)] .
$$

Moreover,

$$
d(T x, T y)=\frac{1}{2} \leq 1=d(x, y),
$$

which deduces that $T$ nonexpansive. It now follows from Theorem 4.4 that $T$ has a best proximity point which is a fixed point in this case and this point is $x^{*}=\frac{1}{2}$.

Acknowledgements. The author would like to thank the reviewer for his/her suggestions.

\section{References}

[1] A. Abkar, M. Gabeleh, Proximal quasi-normal structure and a best proximity point theorem, J. Nonlin. Convex Anal., 14 (2013), 653-659.

[2] A.A. Eldred, W.A. Kirk, and P. Veeramani, Proximal normal structure and relatively nonexpansive mappings, Studia Math. 171 (2005), 283-293.

[3] A. Eldred, V. Sankar Raj, P. Veeramani, On best proximity pair theorems for relatively u-continuous mappings, Nonlin. Anal. 74 (2011), 3870-3875.

[4] R. Kannan, Some results on fixed points. II, Amer. Math. Month., 76 (1969), 405-408.

[5] M. A. Petric, Best proximity point theorems for weak cyclic kannan contractions, Filomat, 25:1 DOI 10.2298/FIL1101145P (2011)

[6] M. Gabeleh, Minimal sets of noncyclic relatively nonexpansive mappings in convex metric spaces, Fixed Point Theory, to appear.

[7] V. Sankar Raj, P. Veeramani, Best proximity pair theorems for relatively nonexpansive mappings, Applied General Topology, 10 (2009), 21-28.

[8] T. Shimizu, W. Takahashi, Fixed points of multivalued mappings in certian convex metric spaces, Topological Methods in Nonlin. Anal., 8 (1996), 197-203. 
[9] P. V. Subrahmanyam, Completeness and fixed-points, Monatshefte fur Mathematik, 80 (1975), 325-330.

[10] W. Takahashi $A$ convexity in metric space and nonexpansive mappings, KODAI MATH. SEM. REP, 22 (1970), 142-149.

[11] C. Wong, Close-to-normal structure and its applications, J. Funct. Anal., 16 (1974), 353-358.

Moosa Gabeleh,

Department of Mathematics,

Ayatollah Boroujerdi University,

Boroujerd, Iran.

Email: gab.moo@gmail.com, Gabeleh@abru.ac.ir 